\title{
In den Schlagzeilen ist es einsam
}

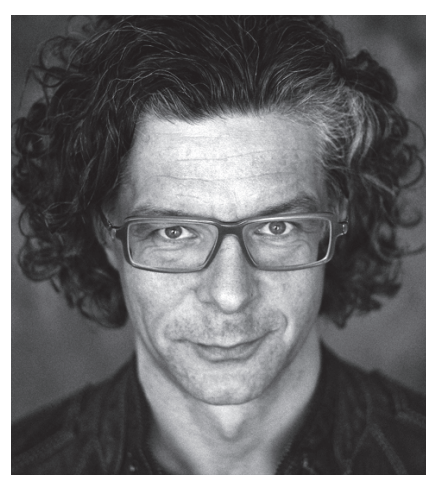

VON CARSTEN TESCH

Carsten Tesch ist Journalist und Moderator beim Kulturradio des Mitteldeutschen Rundfunks.

Zudem berät er soziale Organisationen bei der Medienarbeit und bei der Organisationsentwicklung sowie als Coach in PR-Krisen und Konfliktsituationen. Info@Tesch-Texter.de

\author{
In tatsächlichen oder vermeintlichen Skandalen bei \\ sozialen Organisationen gibt es wenig Solidarität in \\ der Wohlfahrt. Ein Krisen-Netzwerk könnte da hilfreich \\ sein. Eine Runde von Experten aus Wohlfahrt und \\ Hochschulen, die bereit sind, in Krisensituationen in die \\ Öffentlichkeit zu gehen. Denn: In den meisten PR-Krisen \\ steckt die Chance, die akkumulierte Aufmerksamkeit \\ von Politik, Presse und Öffentlichkeit zu nutzen.
}

Wenn soziale Unternehmen und Organi-
sationen mit Konfliktberichterstattung
konfrontiert sind, gibt es regelmäßig
einen Entsolidarisierungseffekt bei den
Kollegen. Wenn dringend Stimmen ge-
braucht werden, die Differenzierung und
realistische Perspektiven böten, ist selten
jemand für Stellungsnahmen zu errei-
chen. Von ihren eigenen Verbänden ist
wenig Unterstützung zu erwarten. Von
den Wettbewerbern schon mal gar nicht.
Dabei könnte ein Krisen-Netzwerk der
Wohlfahrt viel bewirken - für die kon-
kret Betroffenen, vor allem aber für das
Bild, was die Soziale Arbeit insgesamt in
der Öffentlichkeit abgibt.

Seit über zehn Jahren stelle ich diese Frage immer wieder. Ob es um Pflegeskandale, Insolvenzen, sexuellen Missbrauch, Untreuevorwürfe geht, Ausschreibungen für Einrichtungen, Mittelkürzungen oder um Konflikte mit Angehörigen oder Eltern. Gibt es jemand, der nicht in den Konflikt verwickelt ist, der sich mit dem Thema auskennt, dessen Stimme Gewicht hat? Dessen Stellungnahme den Fall um Horizont und Zusammenhänge erweitern würde? Regelmäßig müssen Einrichtungsleiter, Geschäftsführer, Vorstände bei dieser Frage passen.

Wenn man mit der Presse Probleme bekommt, ist man schnell allein. Am schnellsten sind die Politiker weg, die sich gestern gerne noch bei der Grundsteinlegung oder beim Praktikum in der
Einrichtung fotografieren ließen. Eh man sich versieht, stehen die Sozialpolitiker, die eben noch auf der Fachtagung unsere innovativen Projekte gelobt haben, mit Forderungen nach mehr Kontrollen in der Zeitung.

Das Konstruktivste, was die Verbände auf der Regional- und der Bundesebene beitragen, ist, auf die Zuständigkeit der Träger vor Ort zu verweisen. Dann haben wir es zumindest nicht mit Statements von schwarzen Schafen und dringender Aufklärung zu tun. Nicht selten meldet sich dann aber auch noch die Nachbargliederung aus dem eigenen Verband, die noch eine Rechnung offen hat, um abzugrenzen, zu vermuten, zu kritisieren und lange schon gewarnt zu haben.

\section{Solidarität ist selten}

Die Gemeinnützigen nehmen Anteil an den Krisen in der Wohlfahrt, an den Krisen ihrer Kollegen, aber sie solidarisieren sich so gut wie nie. Froh, dass es einen nicht selbst getroffen hat. Kopfschüttelnd über das Krisenmanagement der Anderen. Nicht selten, hab ich den Eindruck, teilen sie die moralische Erregung, auf die die Berichterstattung hinausläuft. Obwohl sie aus der eigenen Erfahrung wissen, dass die Fragen von Schuld und Verantwortung selten so eindeutig sind, wie sie in den Medien erscheinen. 
Einige Beispiele, um zu illustrieren, was ich meine. Kürzlich im Saarland der Fall der beiden Pfleger. Offenbar handelte es sich um einen Kriminalfall. Trotzdem wird die Mitverantwortung des »Systems Pflege" thematisiert. Prompt wird der Pflegeexperte Claus Fussek als Zeuge aufgerufen, dass die Pflege sich prinzipiell gegen Transparenz wehrt. Und der Chef der deutschen HospizStiftung, Eugen Brysch, fordert Schwerpunktstaatsanwaltschaften, um Gewalt in der Pflege zu verfolgen. Warum bleibt das unwidersprochen stehen? Die Wohlfahrt schweigt verunsichert.

Andere Beispiele, wo ich konkret ins Krisen-Management involviert war. Ein großer regionaler Verband geht in die Insolvenz wegen Problemen mit langfristigen Immobilienverpflichtungen. Einem Journalisten fällt auf, dass dieser gemeinnützige Verband gewerbliche Tochterfirmen hat. Es gibt mehrere Artikel, die über das »undurchsichtige Firmengeflecht « spekulieren. Obwohl der Autor bestens von uns mit Informationen über steuerliche Organschaft usw. versorgt ist, bleibt er bei dem Erzählmuster vom »Geflecht«. Hilfreich wäre gewesen, wenn ein LIGA-Präsident mal erklärt hätte, wieso solche gewerblichen Unternehmen inzwischen nötig sind. Und wie viel Sozialpolitik und öffentliche Verwaltung beispielsweise in Kostenverhandlungen dazu beitragen, dass diese vermeintlich effizientere Ökonomisierung der Sozialen Arbeit stattfindet.

Anderes Beispiel. Ein regionaler Träger tritt in einem Ausschreibungsverfahren um eine Klinik gegen große Krankenhauskonzerne an. Wir versuchen, die gemeinnützige Alternative in die öffentliche Diskussion zu bringen. Tatsächlich eine Konzeption mit vielen guten Gründen für eine regionale NonProfit-Struktur gegen den Trend zu Konzentration und Kommerzialisierung im Gesundheitswesen. Wir finden zwar Experten bei anderen gemeinnützigen Trägern, die selbst nicht im Verfahren mitbieten, aber keinen, der für diese Argumente in die Bütt steigt.

\section{Vorschlag: ein Krisen-Netzwerk}

Die Reihe der Beispiele ließe sich fortsetzen. Die Pointe aber ist, auch wenn es sich nach banaler Ratgeberliteratur anhören mag, in den meisten PR-Krisen steckt die Chance, die akkumulierte Aufmerksamkeit zu nutzen. Etwa um Anliegen in die Diskussion einzuspeisen, die es sonst kaum auf die Agenda schaffen.

Ein Krisen-Netzwerk über die Grenzen der Verbände hinweg könnte da hilfreich sein. Eine Runde von Experten aus Wohlfahrt und Hochschulen, die bereit sind, in Krisensituationen in die Öffentlichkeit zu gehen. Und zwar nicht, um das an dieser Stelle auch klar zu sagen, um Fehler und Vergehen schönzufärben, sondern um die simplen Wahrheiten, die Journalisten produzieren (müssen), um eine aufklärerische Komplexität der Aspekte zu bereichern.

Es gibt die Theorie in der Medienwissenschaft, dass Berichterstattung meistens bestimmten Mustern (Frames) folgt. Die beiden wichtigsten Frames im Zusammenhang mit der Sozialen Arbeit sind: Sozial steht drauf, aber drin ist doch nur Geschäft. Nennen wir es das Wasser-Wein-Muster. Und der bereits erwähnte Kontroll-Frame, der für die Pflege aufgerufen wird, aber auch für den Kinderschutz in der Jugendhilfe oder die Mittelverwendung im Zuwendungsbereich. Diese Muster sind, scheint es, unmittelbar an Gefühle gekoppelt. Also wenn der Chef der Treberhilfe in Berlin im Maserati herumfährt, dann ist da viel Gefühl drin für die veröffentlichte Meinung. Auf den ersten Blick ein klarer Fall von einem Wasser-WeinWiderspruch.

\section{Krisen-Kommunikation stellt einfache Erklärungsmuster infrage}

In der Krisen-Kommunikation helfen die Muster, diese und weitere, sich die Perspektive der Journalisten zu vergegenwärtigen. Systemtheoretisch inspiriert würde man sagen, wir Journalisten suchen den Unterschied, der einen Unterschied macht.

Interessant ist, dass der Unterschied gar nicht neu sein muss. In der Pflege zum Beispiel erzählt uns der Medizinische Dienst der Krankenkassen alle Jahre wieder von den vielen verhungerten und vertrockneten Bewohnern. Das ist immer derselbe Unterschied zwischen Hilfeversprechen und Vernachlässigung. Die Pflegekassen präsentieren sich damit paradoxerweise als außerhalb des Systems stehend, als Anwalt der Bewohner. Regelmäßig werden diese zweifelhaften
Erhebungen als skandalöse Wahrheit berichtet.

In der Krisen-Kommunikation geht es meistens darum, auf diese Verkürzungen zu reagieren, in dem wir eine Kommunikation entwickeln, die über diese Muster hinausgeht. Der Skandal braucht Eindeutigkeit. Ein Zuviel an Informationen, die außerdem die unangenehme Eigenschaft haben, widersprüchlich zu sein, lässt die Luft raus.

Nehmen wir noch mal das Beispiel Elversberg im Saarland. Erst mal scheint alles ganz einfach. Zwei Pfleger misshandeln Menschen, denen sie helfen sollten. Da ist ganz klar ein Unterschied zwischen ihrer vorgeblichen Rolle und ihrem tatsächlichen Handeln. Das ist aus journalistischer Perspektive die Geschichte. Relevanter und damit skandalöser (empörender) wäre die Story, wenn man von den beiden auf viele Pfleger schließen könnte. Oder auf die Träger, die diese Pfleger kontrollieren sollten. Mehrere Artikel über Elversberg gehen diesen Schritt, indem sie die zwei "Zeugen« aufrufen (s.o.). Der Bericht in der »Welt « etwa legt nahe, dass es viele solcher grausamen Pfleger geben mag, dass die Träger sie aber nicht kennen oder kennen wollen. Darum brauche es Schwerpunktstaatsanwaltschaften (!).

Wäre es nicht hilfreich gewesen für eine Öffentlichkeit, die sich ohnehin schon so sehr vor ihren Altenheimen fürchtet, wenn relativierende Statements diese Verkürzungen erschwert hätten. Warum gibt es niemand von den benachbarten Trägern, von der LIGA im Saarland, von einer Hochschule, der als unabhängiger Experte die Gelegenheit nutzt, die Kontrollillusionen $\mathrm{zu}$ thematisieren, mit denen die Pflegekassen ihre dominierende Stellung im System begründen. Mit denen Pflege unter einen Generalverdacht gestellt wird. Die Pflege entmündigen. Die in ihren wirklichkeitsfremden Kontrollritualen dazu beitragen, dass Parallelstrukturen entstehen, die sich der Kontrolle schließlich wieder entziehen?

Die Aufmerksamkeit für diese beiden Pfleger wäre eine Gelegenheit gewesen. Aber die Kollegen in der Wohlfahrt waren vermutlich vor allem froh, dass diese beiden nicht ihre eigenen Mitarbeiter waren. 